\title{
Single-side electron multipacting at the photocathode in rf guns
}

\author{
Jang-Hui Han* and Klaus Flöttmann \\ Deutsches Elektronen-Synchrotron, Notkestrasse 85, 22607 Hamburg, Germany \\ Walter Hartung \\ National Superconducting Cyclotron Laboratory, Michigan State University, East Lansing, Michigan 48824, USA
}

(Received 18 October 2007; published 4 January 2008)

\begin{abstract}
Multiple electron impacting (multipacting) can take place in rf fields when the rf components are composed of materials with a secondary electron yield greater than one. In $\mathrm{rf}$ gun cavities, multipacting may change the properties of the vacuum components or even damage them. First systematic measurements of the multipacting occurring in a photocathode rf gun were made at the Fermilab/NICADD Photoinjector Laboratory in 2000. The multipacting properties were found to depend on the cathode material and the solenoid field configuration. In this study, we measure the multipacting properties in more detail and model the secondary electron generation for numerical simulation. Measurements and simulations for the photoinjectors at Fermilab and DESY are compared. The multipacting takes place at the photocathode in rf guns and is categorized as single-side multipacting. In a low rf field, the electrons emitted from the cathode area do not leave the gun cavity within one rf cycle and have an opportunity to travel back and hit the cathode. The solenoid field distribution in the vicinity of the cathode changes the probability of electron bombardment of the cathode and makes a major contribution to the multipacting behavior.
\end{abstract}

DOI: 10.1103/PhysRevSTAB.11.013501

PACS numbers: 52.80.Pi, 79.20.Hx

\section{INTRODUCTION}

Multiple electron impacting (multipacting) is an explosive increase of the number of free electrons in an $\mathrm{rf}$ component such as a cathode, rf cavity, or rf coupler. When an electron hits the surface of a material, more than one secondary electron can be released. In an alternating electric field, the secondary electrons can be accelerated and eventually hit one of the surfaces again to produce more secondary electrons. One speaks of multipacting when the process repeats resonantly and the number of electrons increases exponentially [1-4]. In a general sense, single-side multipacting occurs when the electron hits the wall in a later rf cycle at the same rf phase as the starting phase and near the same location where it has been released. Herein, a type of single-side multipacting is introduced in which the electrons hit the same surface repeatedly, but not always at the same rf phase, with a number of rf cycles between impacts. Multipacting can be initiated by field-emitted electrons, and may lead to vacuum breakdown, rf power loss, or damage to rf components, e.g., the surface of the photocathode. However, the multipacting may also be a useful process for cleaning of the cathode, cavity, or coupler surface [5,6], since the electron bombardment is accompanied by desorption of surface adsorbates.

Multipacting depends on the field configuration, the cavity geometry, and the secondary electron yield (SEY)

\footnotetext{
*Present address: Diamond Light Source, Harwell Science Campus, Didcot, OX11 0DE, United Kingdom.
}

of the cavity materials. It appears preferably at low rf gradients because the SEY shows a maximum value at an impact energy in the kilo electron volt range. External magnetic or DC electric fields can suppress or enhance multipacting [7]. The SEY depends on the material and surface properties and on the impact energy of the primary electron. The SEY of most metals used in $\mathrm{rf}$ components is below one, except over a small energy range where it is somewhat above one. Multipacting on metal surfaces can hence often be eliminated by decreasing the SEY below one via careful rf conditioning [8].

In an rf photocathode gun, a photocathode emits an electron bunch when illuminated by a pulse from a drive laser, and the electron bunch is accelerated by the rf field. In photocathode rf guns used as electron sources for vacuum ultraviolet or x-ray free electron lasers (FELs), semiconductor photocathodes are often employed because the high quantum efficiency $(\mathrm{QE})$ of these materials relaxes the requirements for the drive laser, especially in the case of high average current applications [9]. The figures of merit which characterize the photocathode performance are the operative lifetime, the achievable current density, the extracted charge, the QE, and the uniformity of the emissive layer [10]. At the Fermilab/NICADD Photoinjector Laboratory (FNPL) $[5,6]$ and the photoinjector test facility in Zeuthen (PITZ) [11], cesium telluride $\left(\mathrm{Cs}_{2} \mathrm{Te}\right)$ is used for the photoemissive layer of the photocathodes [9]. $\mathrm{Cs}_{2} \mathrm{Te}$ is less sensitive to gas exposure than other alkali semiconductors, it can generate a high current density electron bunch when deposited on a metallic substrate, and it can also provide a reasonable $\mathrm{QE}$, of order $1 \%$, for 
several months under rf gun operating conditions. The high $\mathrm{QE}$ is in general accompanied with a high SEY which cannot be efficiently reduced by rf conditioning [12].

A set of solenoids surrounding the gun cavity is used to minimize the space-charge-induced emittance growth in the so-called emittance compensation scheme [13]. To produce a low beam emittance, the solenoidal field at the cathode surface must be zero; otherwise there is a contribution to the emittance from the angular momentum generated by the solenoid. The zero field condition is often realized using a bucking solenoid behind the gun: the bucking solenoid produces a magnetic field which opposes the main solenoid field. On the other hand, a nonzero solenoid field at the cathode is required in the so-called flat beam configuration [14-16]. In this case, the angular momentum of the beam is used in a transformation to produce a beam with large emittance ratio $\varepsilon_{x} / \varepsilon_{y}$.

A dependence of the dark current generation and the photocathode QE on the solenoid configuration was measured during flat beam experiments at FNPL in 2000. The authors believe that these observations are related to the occurrence of multipacting in the gun and hence the results are summarized in the first section of this article. A set of dedicated multipacting measurements was performed at PITZ [17]; these are presented in the second section. A model to simulate the multipacting in rf guns is developed in the third section. In the fourth section, simulation results for different solenoid configurations, including the FNPL configurations, are presented.

\section{MEASUREMENTS AT FNPL}

The FNPL gun is schematically shown in Fig. 1. The setup consists of a 1.5 cell copper $(\mathrm{Cu})$ cavity, a side rf coupler to the full cell of the cavity, three solenoids, a

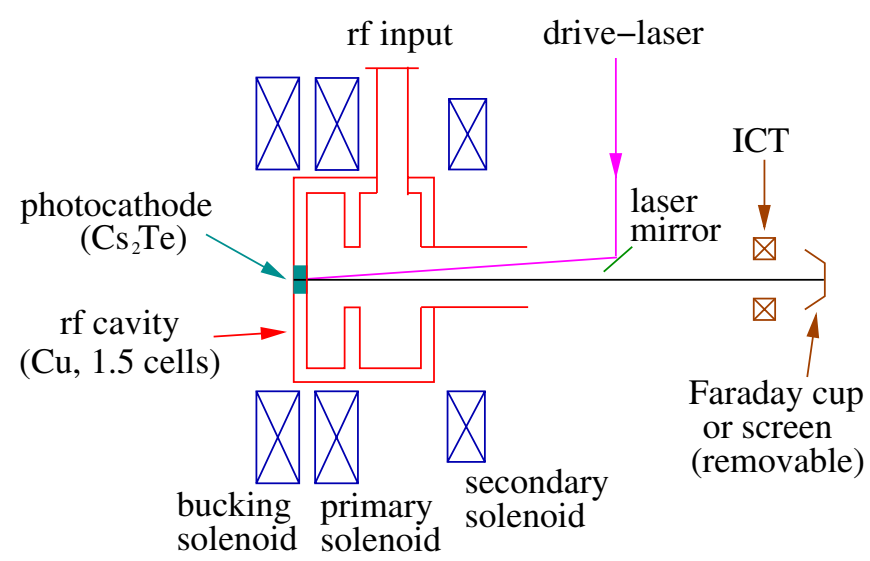

FIG. 1. (Color) Schematic view of the FNPL gun. The rf power for electron beam acceleration is fed into the gun through the side coupler connected to the full cell of the gun cavity. Three solenoids focus the electron beam in order to preserve the emittance. The primary and secondary solenoids have the same polarity, opposite that of the bucking solenoid.
$\mathrm{Cs}_{2} \mathrm{Te}$ photocathode, a drive laser with a wavelength of $262 \mathrm{~nm}$, and various diagnostics. The gun cavity is operated at a resonant frequency of $1.3 \mathrm{GHz}$ [5].

The rf input coupler transfers the rf power from the klystron through the waveguide into the gun cavity. The coupler is connected to the full cell of the gun cavity. Three solenoids surround the rf gun for compensation of the space-charge-induced emittance growth [13]. The solenoid field distribution at FNPL is shown in Fig. 2, along with the rf field distribution and the gun aperture. The fields were calculated using POISSON and SUPERFISH [18]. For the standard round beam generation, the solenoid field at the cathode must be zero in order to minimize the transverse emittance in both planes. If there is a nonzero solenoid field at the cathode surface, the electron beam emerges from the gun with a net angular momentum, which increases the normalized emittance by $e B r_{\text {rms }}^{2} / 2 m_{0} c$ where $r_{\text {rms }}$ is the rms beam size in the radial direction. The flat beam settings correspond to zero bucking solenoid current. In the flat beam case, the longitudinal magnetic field is about $0.08 \mathrm{~T}$ at the cathode; the maximum field is about $0.11 \mathrm{~T}$ in both cases.

The photocathode preparation system for FNPL was developed at INFN Milano-LASA [9]. The photocathodes are coated in a preparation chamber which is connected to the rf gun via a transfer chamber [19]. The front surface of the cathode plug is coated with the photoemissive $\mathrm{Cs}_{2} \mathrm{Te}$ film. The cathode plug is made from pure molybdenum (Mo) in order to minimize the interaction between the emissive materials and the metal substrate [20]. It consists of a cylindrical rod with a diameter of $16 \mathrm{~mm}$. The edge of the plug is rounded to avoid field enhancement and to suppress field emission. Thin layers of $\mathrm{Te}$ and $\mathrm{Cs}$ are deposited one after the other onto the plug through a

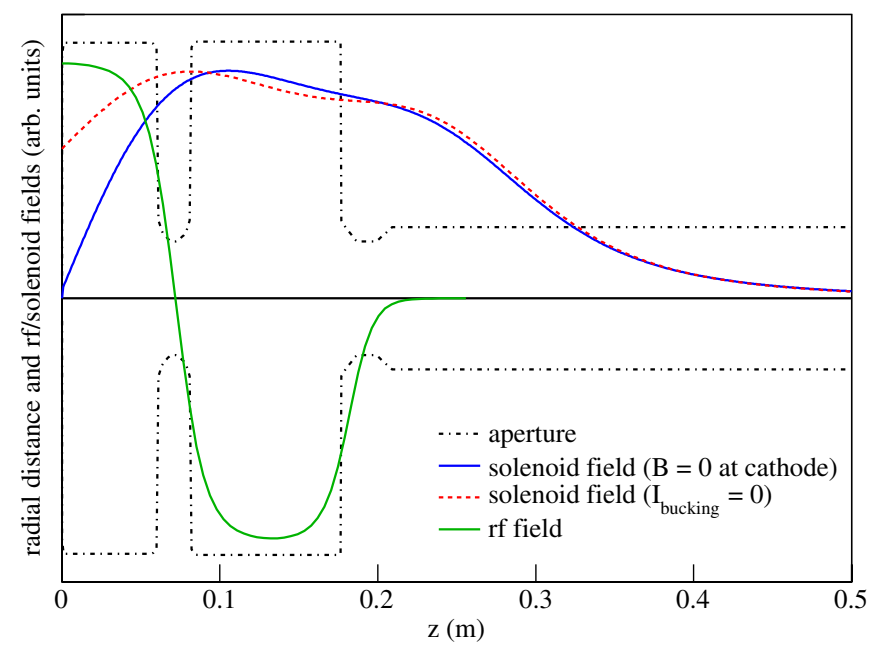

FIG. 2. (Color) Solenoid field, rf field, and simplified aperture for the FNPL gun. As examples, solenoid currents are set as $250 \mathrm{~A}$ for the three solenoids for the case of $B=0$ at cathode. For the $I_{\text {bucking }}=0$ case, $0 \mathrm{~A}, 162 \mathrm{~A}$, and $260 \mathrm{~A}$ are set for the bucking, primary, and secondary solenoids, respectively. 
mask. During the deposition, the cathode plug is heated to $120^{\circ} \mathrm{C}$ so that $\mathrm{Te}$ and $\mathrm{Cs}$ react to produce $\mathrm{Cs}_{2} \mathrm{Te}$. The cathodes must remain in ultrahigh vacuum during and after deposition to avoid oxygen contamination of the photoemissive film.

When changing between the round beam and flat beam solenoid settings, variations in the dark current and QE of the $\mathrm{Cs}_{2} \mathrm{Te}$ photocathode were observed over a time period of several hours $[5,21]$. With the round beam settings ( $B=$ 0 at the cathode), the dark current and QE increased with time. On the other hand, with the flat beam settings $(B \neq 0$ at the cathode), the dark current and QE of the cathode decreased with time. With changing the ratio of the bucking solenoid current to the primary, the characteristics of the multipacting peak were changed [21]. The charge measurements were made with a Faraday cup and an integrating current transformer (ICT).

This effect appears even without the UV drive laser illuminating the cathode, i.e., it is not related to the rejuvenation of the photocathode observed by di Bona et al. [20]. The authors believe that the increase in dark current and QE with round beam settings is caused by a cleaning effect which results from multipacting on the cathode surface. This hypothesis is supported by a number of observations. The multipacting peak (which will be described in detail in the next section) is visible with round beam settings but not with flat beam settings [21]. Moreover, multipacting is not observed with uncoated Mo cathodes in the gun; the dark current of a Mo cathode is independent of time when the solenoid configuration is changed. Finally, the time dependence of the dark current is insensitive to the rf pulse length, which can be explained by the fact that the multipacting occurs only as the rf field increases and decreases at the beginning and the end of the rf pulse.

\section{MEASUREMENTS AT PITZ}

\section{A. Experimental setup}

The PITZ gun is similar to the FNPL gun, except that the PITZ gun has a coaxial rf coupler and two solenoids. The setup for dark current and multipacting measurements is shown schematically in Fig. 3. The gun cavity and rf coupler are designed to be axisymmetric to avoid dipole or higher-order distortions in the cavity field. The rf field profile, shown in Fig. 4, was calculated with MICROWAVESTUDIO [22]. As can be seen in Fig. 4, regions of high surface electric field and likely sources of strong field emission due to the rf field are around the two irises and in the vicinity of the cathode. Numerical simulations show that most of the electrons emitted from the irises hit the cavity aperture and do not escape from the cavity. Field-emitted electrons from the cathode region can be accelerated downstream, depending on both starting location and the starting phase (rf phase at the time of emission) [23].

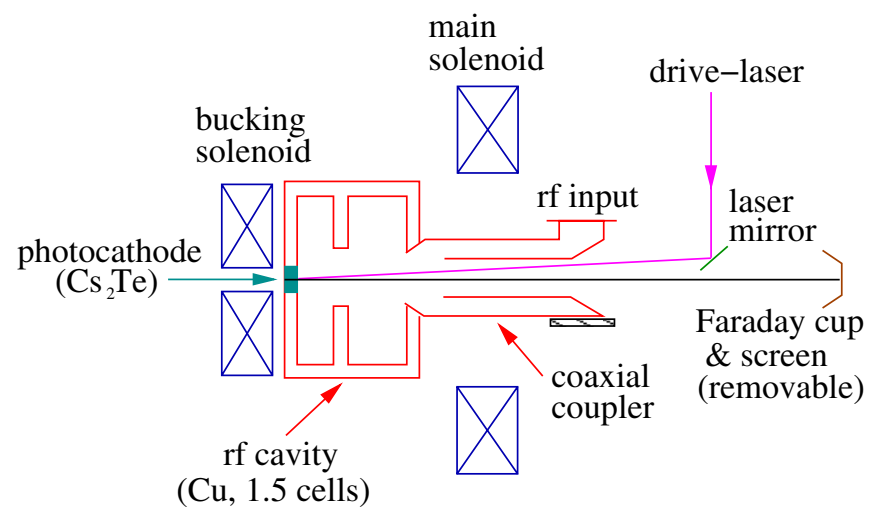

FIG. 3. (Color) Schematic view of the PITZ gun. To ensure axial symmetry for the gun cavity, a coaxial coupler is used. The main solenoid is located further downstream, and therefore the field at the cathode is relatively weak compared to the FNPL case. The bucking solenoid field is concentrated near the cathode only.

The PITZ photocathodes are prepared at INFN MilanoLASA and transported to DESY under ultrahigh vacuum $[24,25]$. The geometry of cathode plugs and the coating procedure are the same as for the FNPL cathodes. The diameter of the $\mathrm{Cs}_{2} \mathrm{Te}$ film is 5 or $10 \mathrm{~mm}$.

Two solenoids surround the gun cavity. The center of the main solenoid is located $0.276 \mathrm{~m}$ downstream of the cathode. The peak field of the main solenoid $B_{z \text {,main }}$ is related to the solenoid current $I_{\text {main }}$ via $B_{z \text {,main }}=5.87 \times 10^{-4} I_{\text {main }}$ [T/A] [26]. The power supply of the main solenoid provides a maximum current of $500 \mathrm{~A}$. A typical operating peak solenoid field is $0.16 \mathrm{~T}$, or a solenoid current of about $280 \mathrm{~A}$ at an rf gradient of around $40 \mathrm{MV} / \mathrm{m}$. Since the main solenoid still produces a nonzero field at the cathode, the bucking solenoid is used for compensation. The maximum in the bucking solenoid field is located $0.037 \mathrm{~m}$ upstream of the cathode and the field opposes that of the main solenoid. The peak field of the bucking solenoid $B_{z, \text { bucking }}$ is related to the current $I_{\text {bucking }}$ via $B_{z \text {,bucking }}=5.33 \times 10^{-4}$ $I_{\text {bucking }}[\mathrm{T} / \mathrm{A}]$ [26]. The bucking solenoid current necessary

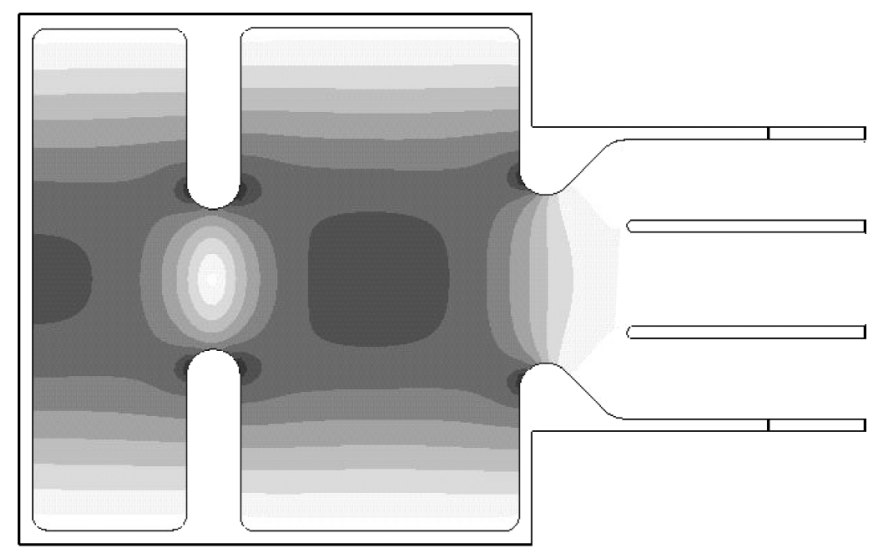

FIG. 4. Contour map of the electric field amplitude in the cavity and part of the coaxial coupler. 


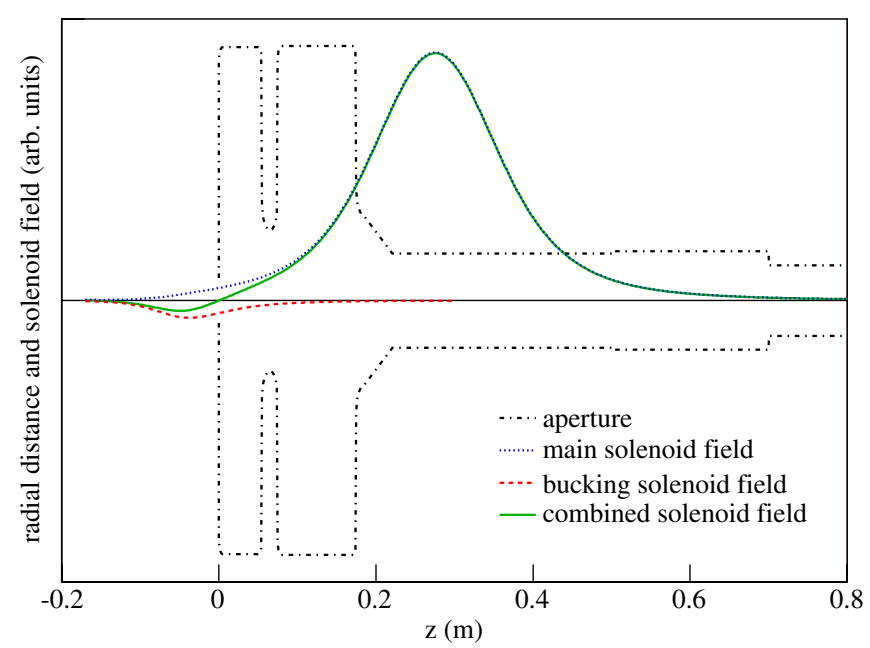

FIG. 5. (Color) On-axis magnetic field distribution for the PITZ solenoids [26] and simplified gun aperture. As an example, solenoid currents are set as $30.5 \mathrm{~A}$ and $400 \mathrm{~A}$ for the bucking and main solenoids, respectively.

for zero magnetic field at the cathode front surface is $I_{\text {bucking }}=0.076 \cdot I_{\text {main }}$. For example, when the main solenoid current is $400 \mathrm{~A}$, a bucking solenoid current of $30.5 \mathrm{~A}$ compensates the main solenoid field at the cathode. With the bucking solenoid turned off, the field at the cathode is 0.012 T. The PITZ gun is not supposed to generate "flat beam" as discussed in the previous section.

The solenoid fields were measured with a Hall probe and the field distributions along the $z$-axis are shown in Fig. 5 [26], along with the gun aperture. The gun aperture is simplified near the rf input port to the gun cavity for the electron tracking simulations. Note that the longitudinal $(z)$ and radial directions of the aperture are scaled separately in Fig. 5.

A Faraday cup located $0.78 \mathrm{~m}$ downstream of the cathode was used for charge measurements in this study. An ICT is installed $0.90 \mathrm{~m}$ downstream of the cathode, but the ICT is not sensitive enough to measure dark current or multipacting electrons since the gain is optimized for the nominal beam charge $(\sim 1 \mathrm{nC})$ and the position is farther downstream than in the FNPL case.

\section{B. Observations}

In rf gun cavities, dark current is generally produced by field emission due to the high rf field for electron beam acceleration. Figure 6 shows an example of dark current measured at PITZ. The main part of the dark current increases slowly with the start of the rf pulse and decreases quickly after the end of the rf pulse because the stored rf field in the cavity has a finite fill/decay time, which is about $2.8 \mu$ s for the $L$-band PITZ cavity, and the field-emitted current $I_{\text {emission }}$ is related to the rf field strength $E$ via $I_{\text {emission }}=C_{1}(\beta E)^{2.5} \exp \left(-C_{2} / \beta E\right)$ [27], where $\beta$ is a field enhancement factor and $C_{1}$ and $C_{2}$ are constants.

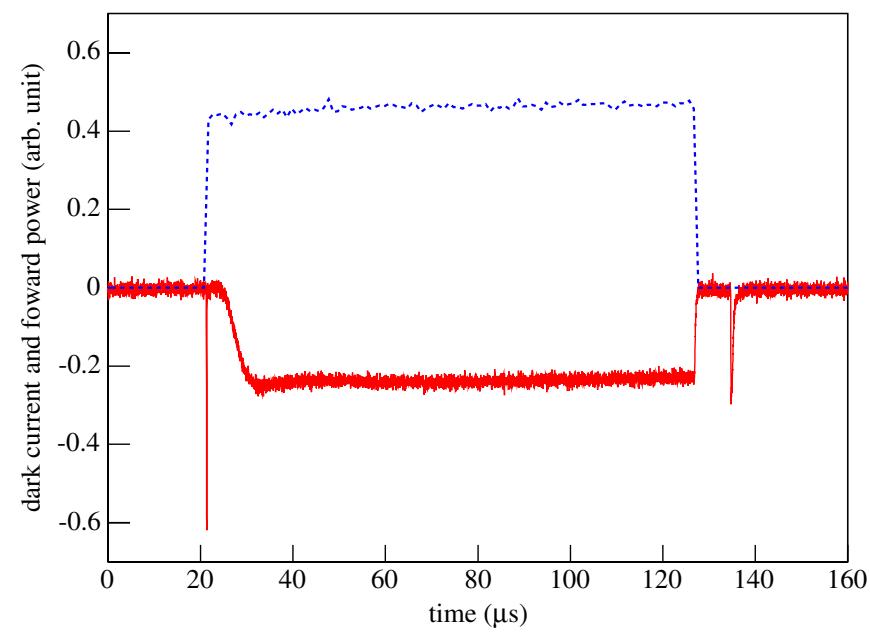

FIG. 6. (Color) A typical trace of dark current from the PITZ gun cavity as a function of time during one rf pulse (full red line). The dark current was measured with the Faraday cup $0.78 \mathrm{~m}$ downstream of the cathode. The rf forward power (dotted blue line) is also shown to indicate the rf pulse length.

Under some operating conditions, two sharp strong peaks appear on the Faraday cup, one at the beginning and the other at the end of the rf pulse; under other conditions, only one of the peaks is present (see Fig. 7 for example). Note that, while the dark current in the plateau scales with the cavity gradient as expected for field emission, the height of the sharp peaks (when they appear) is independent of the cavity gradient. In general, the peaks do not show up for uncoated Mo cathodes (except when the vacuum is very bad) and their behavior is sensitive to the solenoid field configuration.

The front multipacting peak appears immediately after the start of the rf pulse. The rear peak occurs several $\mu \mathrm{s}$ after the end of the rf pulse. These observations imply that the multipacting takes place when the gradient in the cavity is much lower than the gradients used to accelerate the electron bunches. Assuming that the multipacting occurs at the same gradient in the cavity for a certain cathode and solenoid profile, the multipacting condition can be found with the following relation for the front peak:

$$
E_{\mathrm{MP}}=E_{\max }\left[1-\exp \left(-t_{\text {front }} / \tau\right)\right] .
$$

For the rear peak, the corresponding relation is

$$
E_{\mathrm{MP}}=E_{\max } \exp \left(-t_{\text {rear }} / \tau\right),
$$

where $E_{\mathrm{MP}}$ is the rf field at the cathode when the multipacting occurs, $E_{\max }$ is the maximum field of the rf pulse at the cathode, $t_{\text {front }}$ is the delay between the start of the rf pulse and the beginning of the front multipacting peak, $t_{\text {rear }}$ is the delay between the end of the rf pulse and the beginning of the rear multipacting peak, and $\tau$ is the fill/decay time of the rf field in the cavity. Only the rear peak is discussed in the following, because the time difference between the start of the rf pulse and the first multipacting 

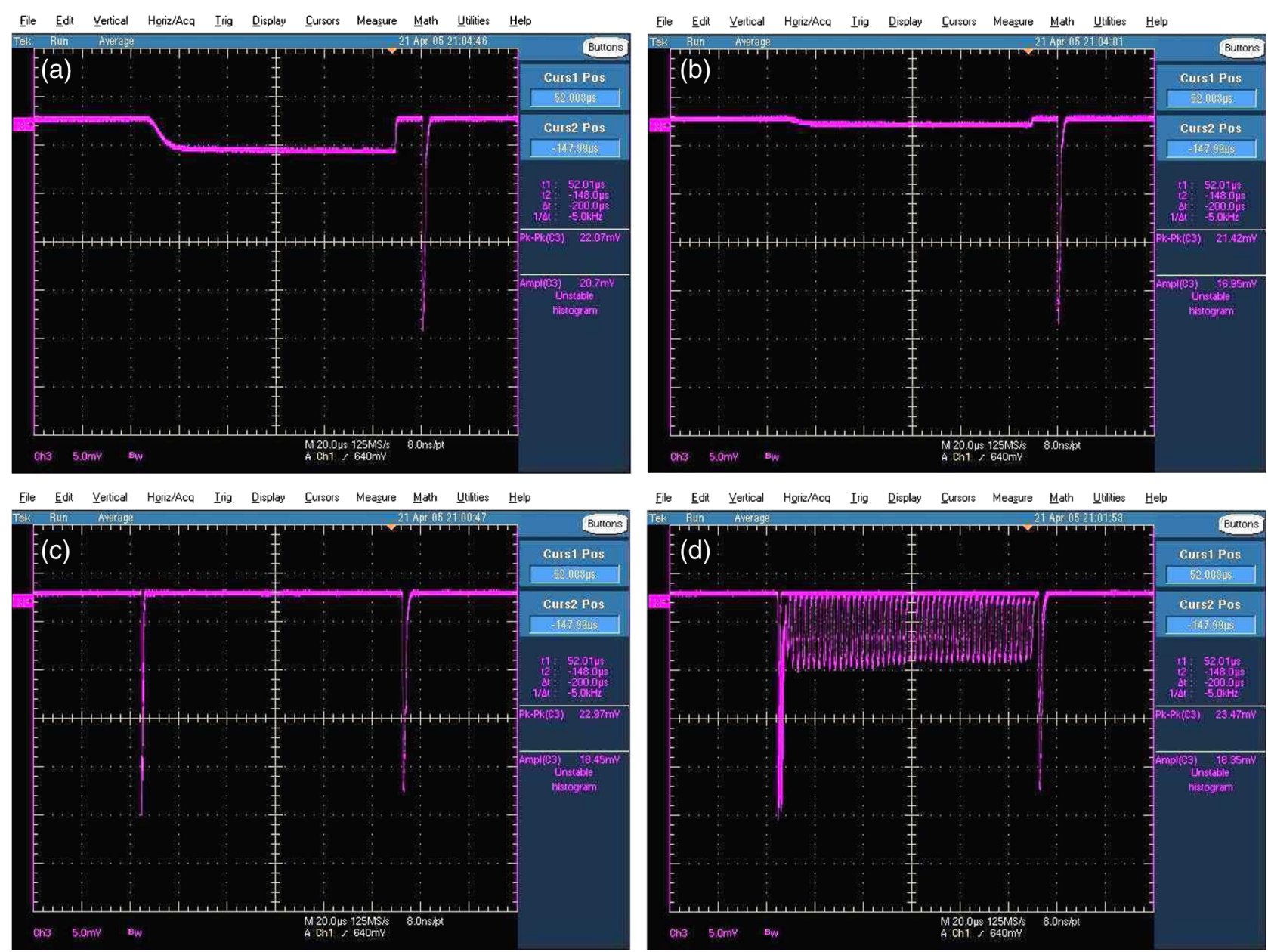

FIG. 7. (Color) Dark current as a function of time measured with the Faraday cup and an oscilloscope for gradients of (a) $40 \mathrm{MV} / \mathrm{m}$, (b) $33 \mathrm{MV} / \mathrm{m}$, (c) $2.7 \mathrm{MV} / \mathrm{m}$, and (d) $2.4 \mathrm{MV} / \mathrm{m}$ on the cathode. The horizontal and vertical scales are $20 \mu$ s per division and $5 \mathrm{mV}$ per division, respectively. Cathode No. 43.2 was used for this measurement when it was fresh (April 2005). The solenoid currents were $400 \mathrm{~A}$ in the main solenoid and $30 \mathrm{~A}$ in the bucking solenoid. At the lowest gradient, multipacting takes place over the entire rf pulse and appears as an oscillation feature.

peak is much less than $1 \mu \mathrm{s}$ and therefore difficult to measure.

The rf field in the cavity is not zero but at a very small background level when the klystron is running but no rf is fed into the klystron. Including the background rf term, Eq. (2) can be rewritten as

$$
E_{\mathrm{MP}}=\left(E_{\mathrm{max}}-E_{\mathrm{BG}}\right) \exp \left(-t_{\mathrm{rear}} / \tau\right)+E_{\mathrm{BG}}
$$

where $E_{\mathrm{BG}}$ is the background rf field strength. From Eq. (3), the delay time can be expressed as a function of $E_{\max }$,

$$
t_{\text {rear }}=\tau \ln \left(E_{\text {max }}-E_{\mathrm{BG}}\right)-\tau \ln \left(E_{\mathrm{MP}}-E_{\mathrm{BG}}\right) .
$$

For operation of the rf gun, the digital signal processing system and the preamplifier of the klystron are adjusted to minimize the background level [28]. The actual background power level is of order $100 \mathrm{~W}$ and is negligible compared to the normal operating power ( $>3 \mathrm{MW}$ ) when discussing the photoelectron beam dynamics. For multi- pacting, however, the background field cannot be neglected.

\section{Dependence on the maximum rf field}

The dependence of the multipacting characteristics on the maximum rf field is represented in Fig. 7. For these measurements, cathode No. 43.2 [24] was used when it was fresh (April 2005). The solenoid currents were $400 \mathrm{~A}$ in the main solenoid and $30 \mathrm{~A}$ in the bucking solenoid, which satisfies the solenoid field compensation condition $(B \approx 0)$ at the cathode. At a maximum rf field of $40 \mathrm{MV} / \mathrm{m}$ [Fig. 7(a)], the rear multipacting peak appears after the main dark current which follows the Fowler-Nordheim equation and tracks the forward rf power pulse. As the maximum rf field decreases, the amplitude of the main dark current decreases [Figs. 7(b) and 7(c)]. However, the height of the multipacting peak does not change visibly [Fig. 7(b)]. As the maximum rf field decreases further, the front multipacting peak newly appears [Fig. 7(c)]. To 
initiate the multipacting, a sufficient number of free, i.e., field-emitted, electrons are required, because only those electrons which hit the cavity wall at the right phase and with the right energy can produce secondaries and meet the resonance condition. Since field emission depends strongly on the gradient, the rear peak, which starts at a higher field and extends to smaller field levels, is more stable than the front peak which has to start at the lower field level. When the cavity is operated at a lower gradient, the cavity field increases more slowly, so multipacting can occur more easily at the beginning of the rf pulse.

When the maximum rf field decreases below a certain threshold value, multipacting occurs over the entire duration of the rf pulse with an oscillatory behavior [Fig. 7(d)]. At this low power level, the beam loading induced by the multipacting might reduce the rf field strength in the gun cavity as the number of free electron increases. When the field level is too low, the multipacting condition is no longer satisfied, and the multipacting stops until the rf field strength is sufficiently high again.

The position and the amplitude of the rear multipacting peak were observed as a function of the maximum rf field

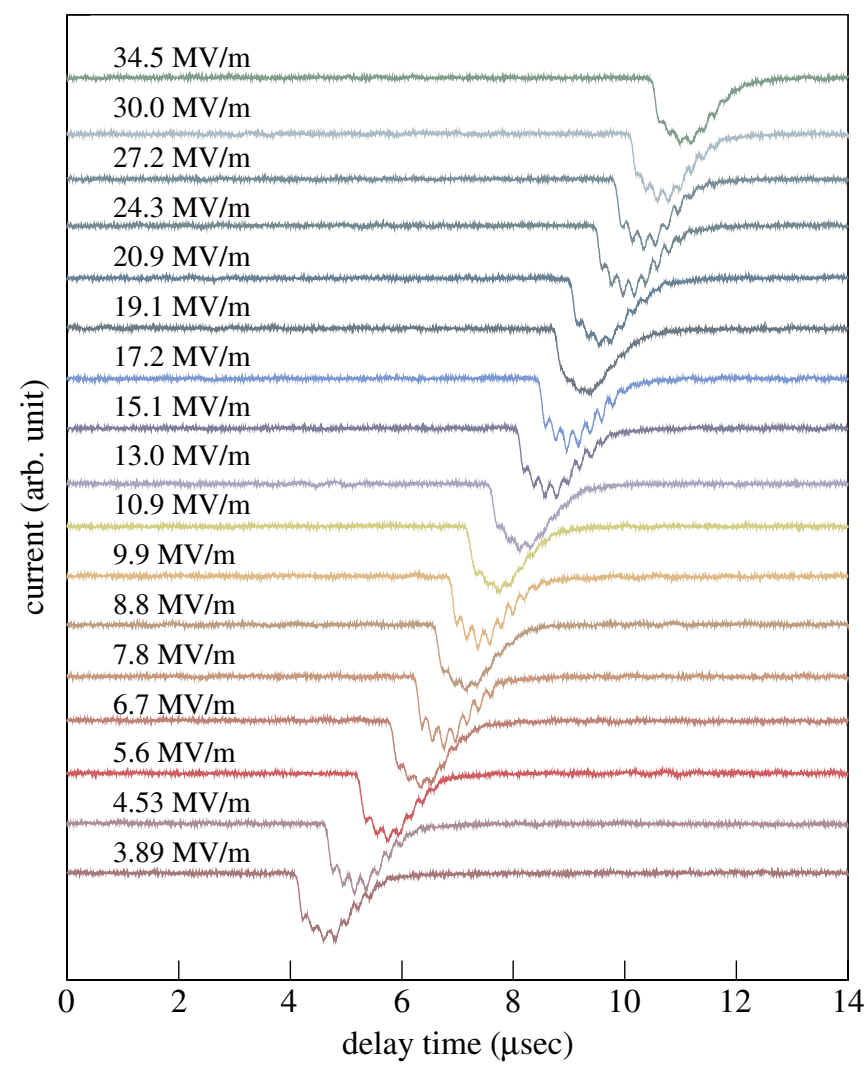

FIG. 8. (Color) Faraday cup signal as a function of the time elapsed since the end of the rf pulse for different values of the maximum rf field at the cathode. The delay $\left(t_{\text {rear }}\right)$ between the end of the rf pulse and the start of the rear multipacting peak decreases as the maximum rf field decreases. The peak amplitude of the multipacting current spike does not change visibly with respect to the maximum rf field.

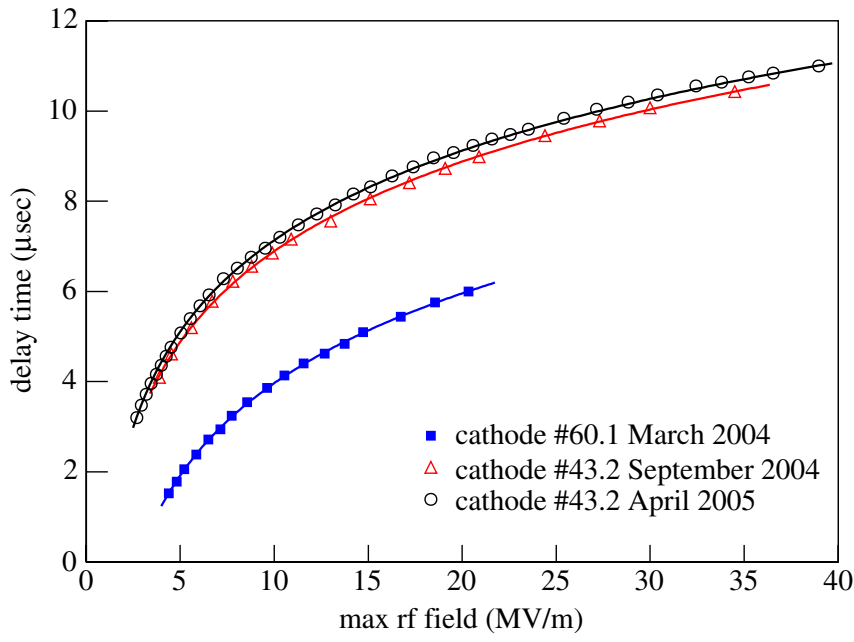

FIG. 9. (Color) Measured delay time $\left(t_{\text {rear }}\right)$ between the end of the rf pulse and the beginning of the multipacting spike as a function of the maximum rf field amplitude for two cathodes (No. 60.1 and No. 43.2 [24]), with fits to Eq. (4). The solenoid currents for these measurements were $400 \mathrm{~A}$ in the main solenoid and $30.5 \mathrm{~A}$ in the bucking solenoid.

in the cavity, as shown in Fig. 8. The delay time decreases as the maximum rf field is lowered, but the shape and strength of the multipacting peak does not change. This result shows that the multipacting process is independent of the maximum rf field. As the rf field decays, the electron multiplication always takes place at a particular rf field amplitude.

Three series of measurements were done on two different cathodes, and the results are shown in Fig. 9, along with fits. In each case, $\chi$-square fits were done using Eq. (4) to find the multipacting field $E_{\mathrm{MP}}$, the rf background $E_{\mathrm{BG}}$, and the fill/decay time $\tau$. The fit results are summarized in Table I. The two different cathodes show different multipacting fields $E_{\mathrm{MP}}$. Possible reasons for this difference are geometrical differences in the $\mathrm{Cs}_{2} \mathrm{Te}$ films (diameter and thickness) or mechanical damage of the cathodes. The fitted rf fill/decay times are in good agreement with the filling time of $2.78 \mu \mathrm{s}$ obtained from rf measurements on the cavity. Note that the rf fill/decay time may change slightly for different cathodes because the gun's operating temperature is adjusted for different cathodes.

Cathode No. 60.1 shows a smaller delay time, i.e., multipacting starts already at a higher field, but probably extends

TABLE I. Fit results for the multipacting measurements in Fig. 9.

\begin{tabular}{cccc}
\hline \multirow{2}{*}{$\begin{array}{c}\text { Cathode } \\
\text { Measurement date }\end{array}$} & Morch 2004 & \multicolumn{2}{c}{ No. 43.2 } \\
\hline$E_{\mathrm{MP}}(\mathrm{MV} / \mathrm{m})$ & 2.70 & 1.04 & 1.07 \\
$E_{\mathrm{BG}}(\mathrm{MV} / \mathrm{m})$ & 0.36 & 0.18 & 0.31 \\
$(\mu \mathrm{s})$ & 2.80 & 2.83 & 2.83 \\
\hline \hline
\end{tabular}



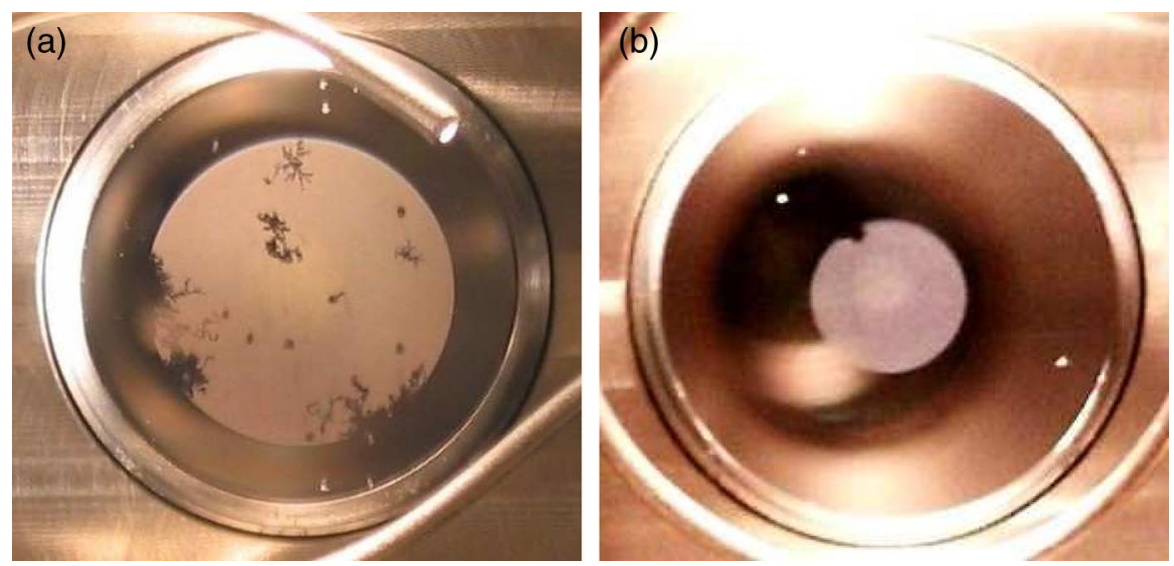

FIG. 10. (Color) Photographs: (a) cathode No. 60.1 in March 2004 and (b) cathode No. 43.2 in February 2005. Cathode No. 60.1 has a $\mathrm{Cs}_{2}$ Te film of diameter $10 \mathrm{~mm}$ and cathode No. 43.2 has a film of diameter $5 \mathrm{~mm}$. The mirrorlike Mo plug surface reflects the images of the viewport and the flange of the vacuum chamber. The metal wire visible in the corners of the picture is the anode installed for QE measurements using a UV lamp.

down to a similar field as for cathode No. 43.2. Figure 10 shows photographs of the two cathodes. The pictures were taken with a digital camera through the viewport of the cathode transport chamber [29]. The cathodes are stored in the cathode chamber under ultrahigh vacuum and can be selected and moved to the gun cavity with an actuator. When the measurements were made, cathode No. 60.1 was partially damaged [Fig. 10(a)] but the central part of the cathode had still emissive material $\left(\mathrm{Cs}_{2} \mathrm{Te}\right)$. Cathode No. 43.2 was fresh except for a small zone of mechanical damage at the upper left corner [Fig. 10(b)]. The damage was not visible when the cathode was coated at INFN Milano-LASA.

\section{Dependence on the solenoid setting}

While the maximum rf field affects only the delay time of the multipacting peak, the solenoid field distribution in the vicinity of the cathode has an impact not only on the delay time, but also on the shape of the multipacting spike and on the charge contained in the spike. Moreover, the multipacting can be completely suppressed for certain solenoid field configurations.

A series of measurements were made with cathode No. 43.2 in November 2004 to measure the dependence of the multipacting behavior on the solenoid field distribution. A maximum rf field of $39 \mathrm{MV} / \mathrm{m}$ at the cathode was used. The delay time for the rear peak was measured as a function of the bucking solenoid current for several main solenoid currents (Fig. 11). The multipacting spike could not be detected with the Faraday cup for bucking solenoid currents between 18 and $26 \mathrm{~A}$ or greater than $35 \mathrm{~A}$. Compensation of the solenoid field at the cathode requires bucking solenoid currents of 24.5 to $30.5 \mathrm{~A}$ for main solenoid currents of 320 to $400 \mathrm{~A}$, i.e., at higher bucking solenoid currents the solenoid field is overcompensated and the point of zero magnetic field is inside the cavity.
As the main solenoid current increases, the delay time becomes shorter for all bucking solenoid settings and the multipacting-free zone shifts to higher bucking solenoid currents. Note that no multipacting could be observed at main solenoid currents below $340 \mathrm{~A}$ in the overcompensated field configuration.

The multipacting field $E_{\mathrm{MP}}$ can be fitted from the measurements shown in Fig. 11 using Eq. (3), under the assumption that the background field $E_{\mathrm{BG}}$ and the fill/decay time $\tau$ remain constant. The results (Fig. 12) show that the multipacting takes place when the rf field at the cathode is less than $1 \mathrm{MV} / \mathrm{m}$.

Results of charge measurements with the Faraday cup are shown in Fig. 13. The charge contained in the multi-

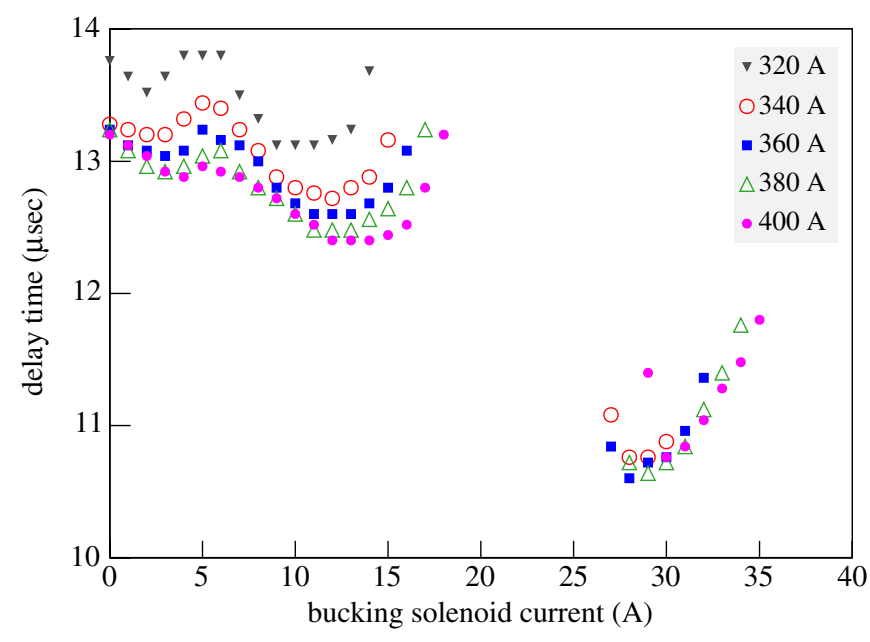

FIG. 11. (Color) Measurements of the delay time $\left(t_{\text {rear }}\right)$ between the end of the rf pulse and the beginning of the multipacting spike as a function of bucking solenoid current $\left(I_{\text {bucking }}\right)$ for several main solenoid currents $\left(I_{\text {main }}\right)$. No multipacting is detected at bucking solenoid currents between 18 and $26 \mathrm{~A}$ or higher than $35 \mathrm{~A}$. 


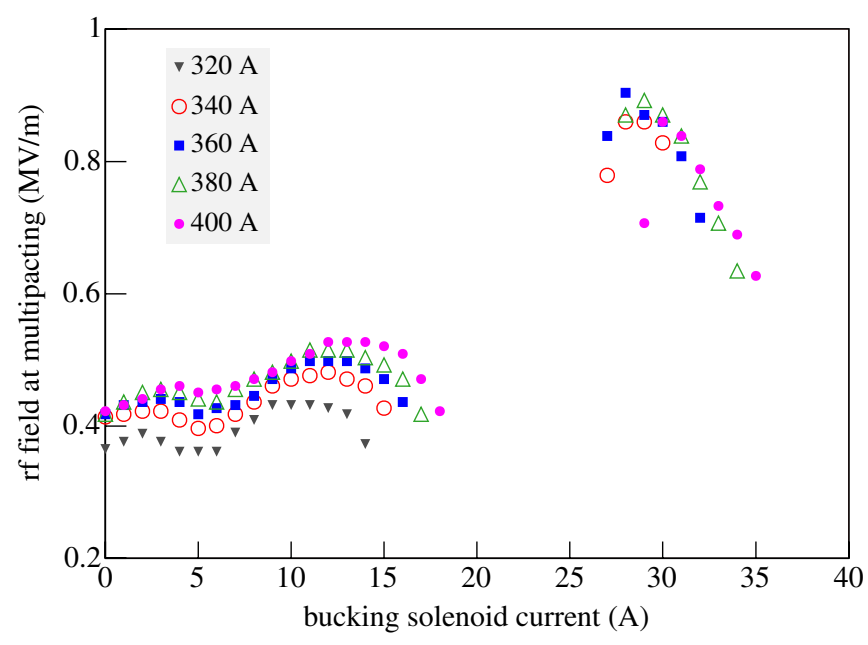

FIG. 12. (Color) Fitted rf field at multipacting $\left(E_{\mathrm{MP}}\right)$ corresponding to the delay time $\left(t_{\text {rear }}\right)$ measurements shown in Fig. 11.

pacting peak reaches values above $1 \mathrm{nC}$. The plots in Figs. 12 and 13 show a similarity in their shapes. Note that there is generally higher charge measured in the region of magnetic undercompensation and lower charge measured in the overcompensation region. The reason for this is unclear. According to the measurements above, multipacting does not take place at the nominal operating conditions for PITZ (a main solenoid current of about 320 A and a bucking solenoid current of about $24 \mathrm{~A}$ at an $\mathrm{rf}$ gradient of $42 \mathrm{MV} / \mathrm{m}$ ) or for the free electron laser at Hamburg (FLASH) [30] (a main solenoid current of about $280 \mathrm{~A}$ and a bucking solenoid current of about $20 \mathrm{~A}$ at an rf gradient of $42 \mathrm{MV} / \mathrm{m}$ ).

Figure 14 shows the variation in the shape of the multipacting peak with the bucking solenoid current for a constant main solenoid current of 400 A. At a bucking solenoid current of $29 \mathrm{~A}$, the peak is narrow, i.e., the range

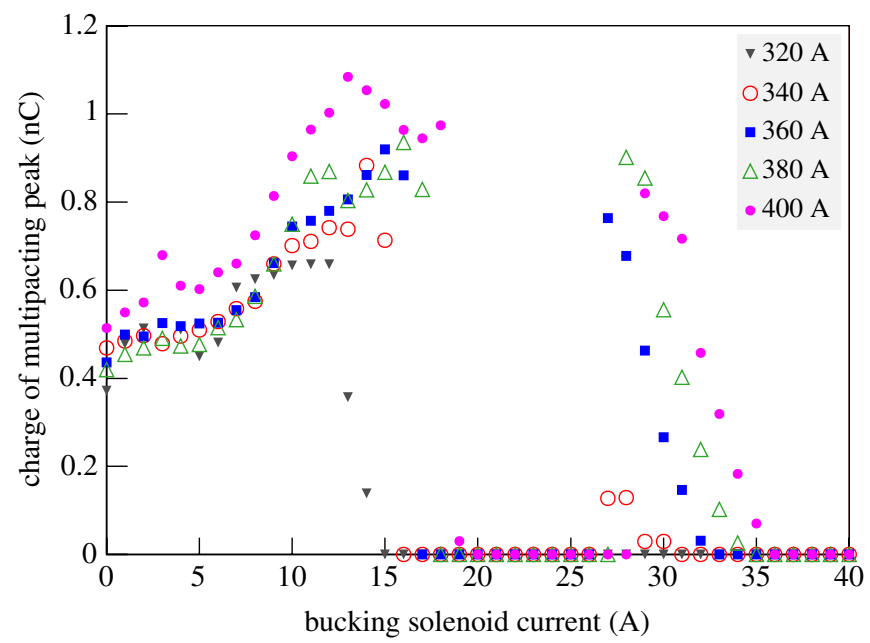

FIG. 13. (Color) Measured charge in the rear multipacting spike as a function of the bucking solenoid current for several main solenoid currents.

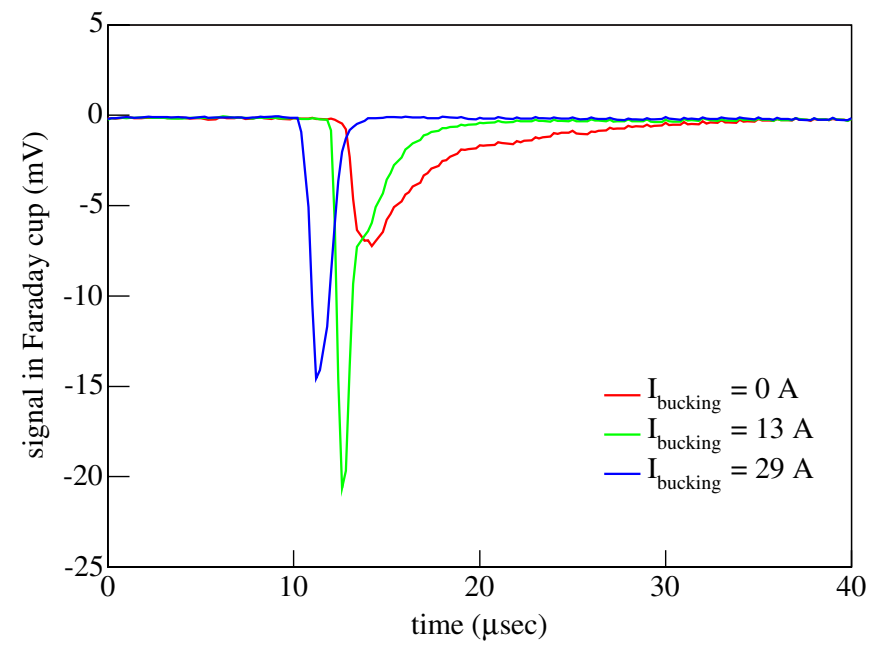

FIG. 14. (Color) Shape of the multipacting peak for several bucking solenoid currents at a main solenoid current of $400 \mathrm{~A}$.

of gradients in which multipacting can take place is small. At zero bucking solenoid current, the peak is wide and the tail extends up to $30 \mu \mathrm{s}$ after the end of the rf pulse, which corresponds to the level of the background field. At $13 \mathrm{~A}$ the charge in the peak is maximal while the width of the peak is between the 29 and 0 A case, i.e., the range of $\mathrm{rf}$ field over which multipacting takes place is intermediate and secondary electron production is the most effective.

The measurements described above were found to be reproducible when repeated within a few hours. Some general characteristics are investigated in the following section via numerical calculations.

\section{MODELING OF MULTIPACTING}

When a primary electron hits the $\mathrm{Cs}_{2} \mathrm{Te}$ photocathode, it may generate secondary electrons because the photocathode has an SEY larger than 1 [12]. As secondary electron emission and photoemission are similar processes, high QE materials have a high SEY [31]. The number of generated secondary electrons is modeled in the tracking program ASTRA [32] according to the relation [33]

$$
\delta\left(E_{p}\right)=\delta_{\max } \frac{E_{p}}{E_{p, \max }} \frac{s}{s-1+\left(E_{p} / E_{p, \max }\right)^{s}},
$$

where $\delta\left(E_{p}\right)$ is the SEY depending on the energy $E_{p}$ of the primary electron, $\delta_{\max }$ is the maximum SEY, which occurs at the primary electron energy $E_{p, \max }$, and $s$ is a fit parameter. The model for production and tracking of secondary electrons is the same as described in Ref. [12]. In this secondary emission model, the incident angle of primary electrons is assumed to be normal to the cathode surface and the time delay of the secondary emission is assumed to be negligibly small.

An example of multipacting trajectories is shown in Fig. 15. In this simulation, a seed electron starts at an $\mathrm{rf}$ 

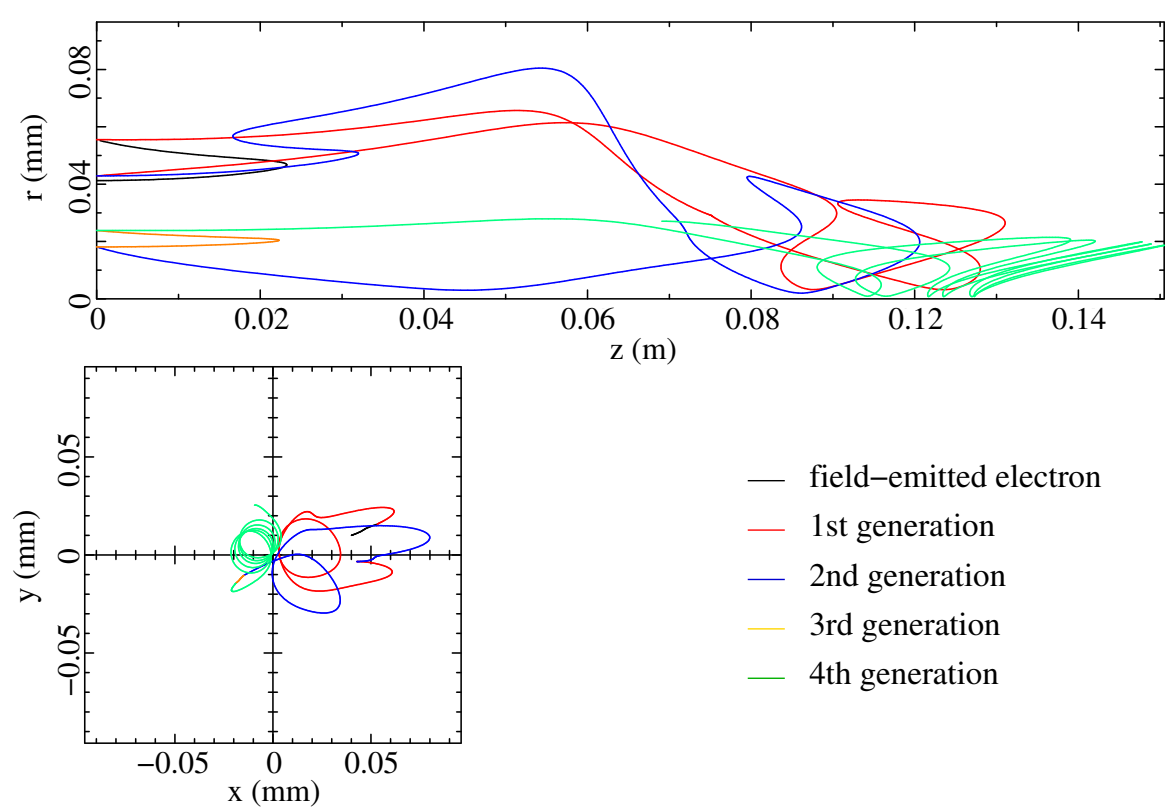

— field-emitted electron

- 1st generation

— 2nd generation

- 3rd generation

— 4 th generation

FIG. 15. (Color) One example of multipacting trajectories calculated with ASTRA for the PITZ gun.

phase of $90^{\circ}$, with an $\mathrm{rf}$ field strength of $5.0 \mathrm{MV} / \mathrm{m}$, a main solenoid current of $400 \mathrm{~A}$, and a bucking solenoid current of $30.5 \mathrm{~A}$. A high rf field is used for ease of visualization of the electron trajectories. The seed electron is field emitted from the cathode with a radial offset of $0.04 \mathrm{~mm}$ from the electrical center of the cavity. If it starts at the right $\mathrm{rf}$ phase, the seed electron hits the cathode again and generates secondary electrons (1st generation). The 1st generation electrons drift for several rf cycles and hit the cathode again to produce 2 nd generation electrons. For a clear illustration, only one secondary electron for each generation is shown in Fig. 15. In reality, depending on the impact energy, multiple electrons may be produced. After several generations of secondary emission, the number of electrons increase exponentially and multipacting takes place. The multipacting occurs at the front surface of the cathode only, therefore it is single-side multipacting.

In the following, the numerical multipacting simulations will be discussed in detail. In the simulations, an electron multiplication factor is defined as the number of "active" electrons per seed electron after a specified number of $\mathrm{rf}$ cycles. An active electron is a secondary electron which is able to produce a next generation of secondary electrons, i.e., which is not lost after impacting on an inactive surface (Mo or $\mathrm{Cu}$ ) or after generating secondary electrons on the $\mathrm{Cs}_{2} \mathrm{Te}$ cathode. In the simulations, the number of rf cycles must be limited in order to limit the calculation time. However, since the electrons can move for many rf cycles before hitting a wall, the calculated multiplication factor has some uncertainty, and the derivation of an exponential growth rate is difficult.

For the following simulations, the secondary emission parameters were chosen as follows: $\delta_{\max }=20, E_{p, \max }=$ $1 \mathrm{keV}$, and $s=2.2$. These values are slightly different from the values used in Ref. [12], but the parameters are expected to depend on the $\mathrm{Cs}_{2} \mathrm{Te}$ film characteristics, which may be modified by damage to the cathode over time.

\section{A. Electron dynamics in an rf field of low strength}

The equation of motion of an electron in an rf field is written as

$$
\frac{d \boldsymbol{p}}{d t}=e \boldsymbol{E}_{0}(\boldsymbol{r}) \cos (\omega t)+\frac{e}{\omega} \boldsymbol{v} \times\left[\boldsymbol{\nabla} \times \boldsymbol{E}_{0}(\boldsymbol{r})\right] \sin (\omega t),
$$

where $\boldsymbol{E}_{0}(\boldsymbol{r})$ is the amplitude vector of the rf field and $\boldsymbol{r}$ is the position vector of the electron. In this study, numerical calculations with ASTRA were done with a 3D rf field profile. The seed electrons start with a uniform transverse distribution over the cathode area of $5 \mathrm{~mm}$ diameter and a given rf phase.

When the maximum rf field is smaller than about $10 \mathrm{MV} / \mathrm{m}$, electrons starting at an emission phase around $90^{\circ}$ have a large phase slippage and can not escape from the cavity any longer. Electrons emitted with starting phases near $90^{\circ}$ either travel back to the cathode, possibly generating secondary electrons, or are trapped by the rf field, drifting back and forth inside the cavity.

Multipacting is generally unlikely without the solenoid field, because the rf field alone is not strong enough to trap the electrons inside the gun cavity. Even though a possible multiplication can be numerically calculated (see Fig. 16), no multipacting has been observed without the solenoid field. In this simulation, 500 seed electrons were generated in order to reduce the statistical error in the random generation of secondary electrons. 


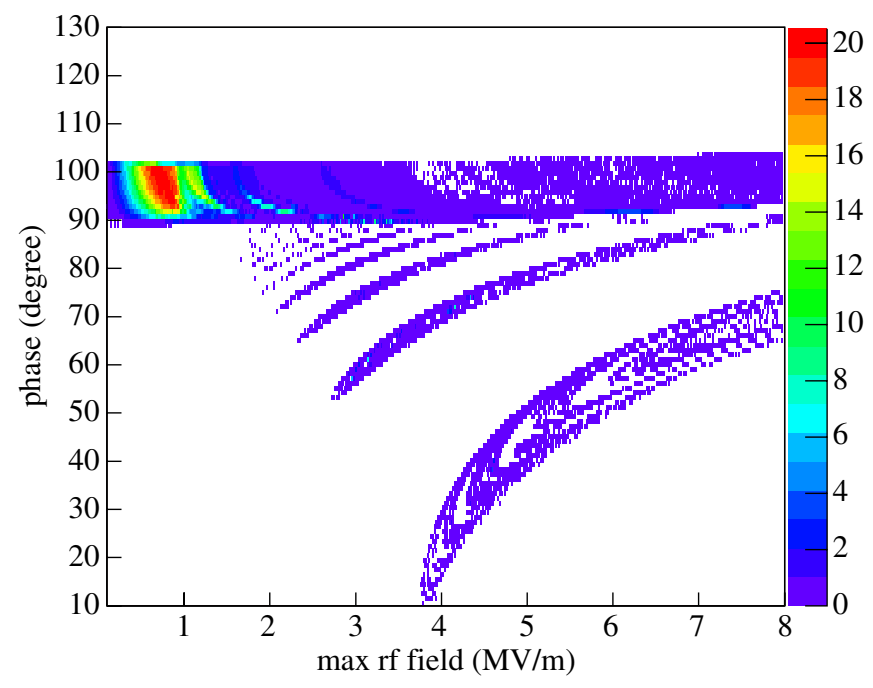

FIG. 16. (Color) Electron multiplication factor as a function of the maximum rf field and the emission phase with no applied solenoid field. The primary and secondary electrons were tracked for $100 \mathrm{rf}$ cycles. For electrons starting at a maximum rf field between 0.1 and $1.8 \mathrm{MV} / \mathrm{m}$ and at an $\mathrm{rf}$ phase between $90^{\circ}$ and $96^{\circ}$, the multiplication factor is higher than 5 for this time period. The white area shows no multipacting condition.

\section{B. Electron dynamics in a solenoid field}

With a solenoid field, the equation of motion of an electron is modified as

$$
\begin{aligned}
\frac{d \boldsymbol{p}}{d t}= & e \boldsymbol{E}_{0}(\boldsymbol{r}) \cos (\omega t)+\frac{e}{\omega} \boldsymbol{v} \times\left[\boldsymbol{\nabla} \times \boldsymbol{E}_{0}(\boldsymbol{r})\right] \sin (\omega t) \\
& +e \boldsymbol{v} \times \boldsymbol{B}_{\mathrm{sol}}(\boldsymbol{r}),
\end{aligned}
$$

where $\boldsymbol{B}_{\text {sol }}(\boldsymbol{r})$ is the inhomogeneous magnetic field generated by the solenoids.

When a solenoid field is applied, electrons in the cavity can change their longitudinal flight direction because of the magnetic mirror effect. The backward force due to the magnetic mirror can be written as [34]

$$
\frac{d p_{z}}{d t}=\frac{e}{2} r^{2} \dot{\phi} \frac{\partial B(z)}{\partial z}
$$

where $\dot{\phi}$ is the angular velocity around the $z$ axis. Because of this backward force induced by the solenoid field, the electrons have a higher probability of returning to the photocathode to produce secondary electrons.

When the bucking solenoid is applied, the magnetic field gradient at the cathode is changed (see Fig. 5). Figure 17 shows simulation results for a solenoid configuration with magnetic field at the cathode compensated by the bucking solenoid. The multipacting zone is larger than in the case without a solenoid field (Fig. 16), especially near $90^{\circ} \mathrm{rf}$ phase where the field emission is strongest.

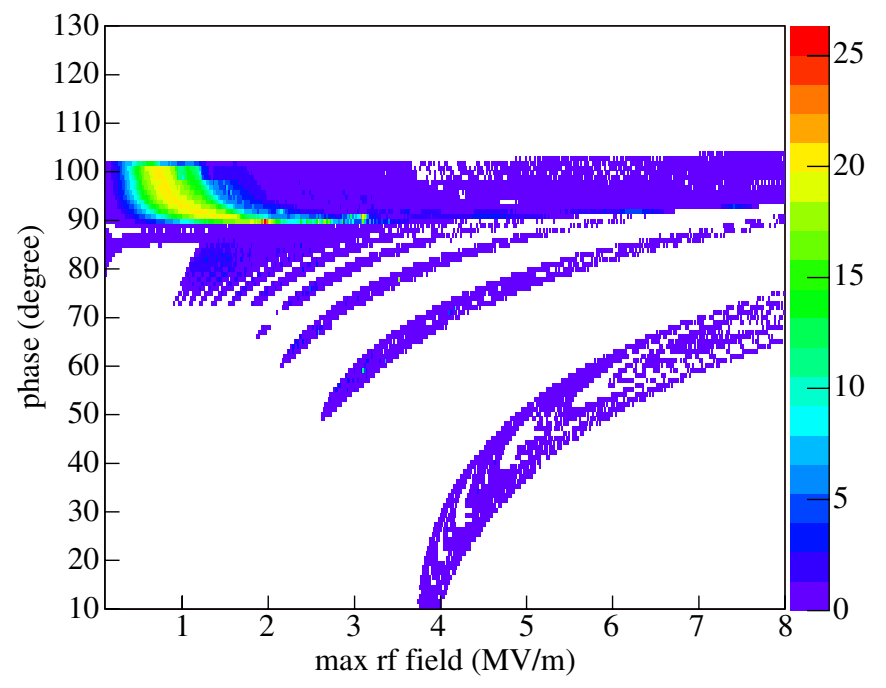

FIG. 17. (Color) Electron multiplication factor as a function of the maximum rf field and the emission phase for main and bucking solenoid currents of 400 and 30.5 A, respectively. Primary and secondary electrons were tracked for $100 \mathrm{rf}$ cycles. The zone of high multiplication factor is larger than in Fig. 16. The multiplication factor is especially high at $\mathrm{rf}$ phases near $90^{\circ}$, where the field emission is highest. The white area shows no multipacting condition.

\section{Multiplication of field-emitted electrons}

Realistic multipacting conditions integrate the effects due to the emission phase and the 3D distribution of the field-emitted electrons, i.e., the 2D transverse and temporal distribution of the seed electrons. For the following results, 1000 seed electrons were started from the cathode area. The temporal profile of the seed electrons formed a Gaussian distribution around the $90^{\circ}$ rf phase with a spread of $14^{\circ}$ ( $\sim 30 \mathrm{ps}$ ) rms. This temporal profile is expected for field-emitted electrons following the Fowler-Nordheim equation in a sinusoidal rf field $[35,36]$. A time corresponding to $140 \mathrm{rf}$ cycles $(\sim 108 \mathrm{~ns})$ was used for the electron tracking; this time was the basis for the calculation of the multiplication factor.

The multiplication factor was calculated as a function of the maximum rf field at the cathode for three different solenoid configurations. The main solenoid current was either 0 or $400 \mathrm{~A}$. At $400 \mathrm{~A}$, the maximum solenoid field is $0.235 \mathrm{~T}$, occurring $0.276 \mathrm{~m}$ downstream of the cathode. The bucking solenoid current was either 0 or $30.5 \mathrm{~A}$. The field of the main solenoid at the cathode position is only $5 \%$ of the maximum (see Fig. 5). Nevertheless, the bucking solenoid has a strong influence on the multipacting. The simulation results in Fig. 18 show that the multiplication is strong for the compensated case $\left(I_{\text {bucking }}=30.5 \mathrm{~A}\right)$ and negligibly weak for the other cases.

In actual operation, the rf field in the gun cavity decays exponentially from $40 \mathrm{MV} / \mathrm{m}$ (typically) to 0 with the $\mathrm{rf}$ fill/decay time. Figure 19 shows the evolution of the multiplication factor with time in a decaying rf field. For this 


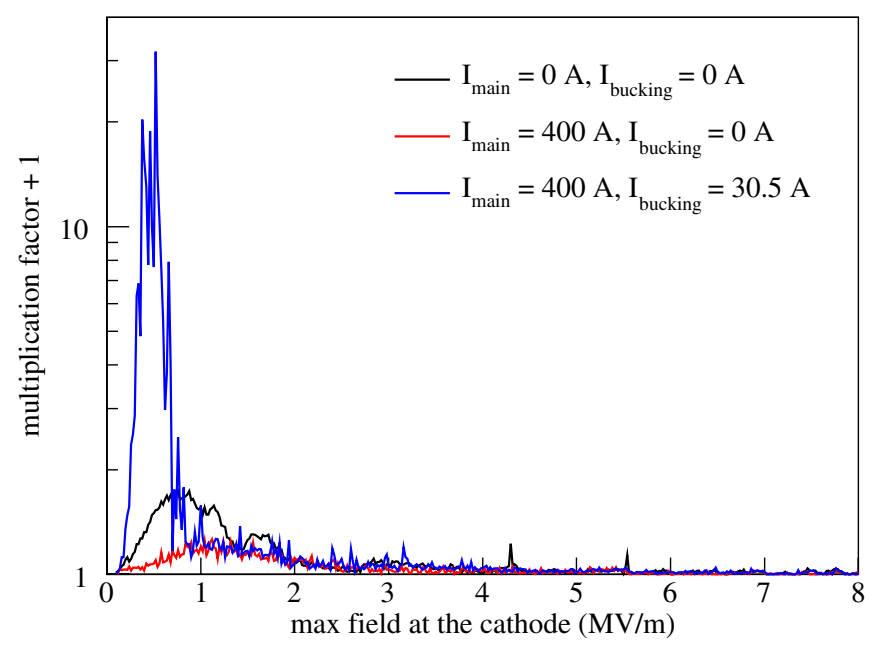

FIG. 18. (Color) Multiplication factor after $140 \mathrm{rf}$ cycles as a function of the maximum field at the cathode for the PITZ gun. Three combinations of main and bucking solenoids currents are shown; 1 is added to the multiplication factor for better visualization on a logarithmic scale.

simulation, 1000 seed electrons were generated and tracked for $300 \mathrm{rf}$ cycles while the rf field decays with a decay time constant of $2.78 \mu \mathrm{s}$, starting at $0.6 \mathrm{MV} / \mathrm{m}$. During the calculation, the field decreases to about $0.55 \mathrm{MV} / \mathrm{m}$. The main solenoid current was $400 \mathrm{~A}$ and the bucking solenoid current was $30.5 \mathrm{~A}$. On average, the simulation shows an exponential increase of the number of electrons. Note, however, that the multiplication takes place in steps separated by many rf cycles. An estimation of the exponential growth rate requires a simulation with more seed particles and more rf cycles, but we are limited by the available computing power.

In Fig. 14, the multipacting peak grows to its maximum value in about $1 \mu \mathrm{s}$. The time of $1 \mu \mathrm{s}$ corresponds to 1300

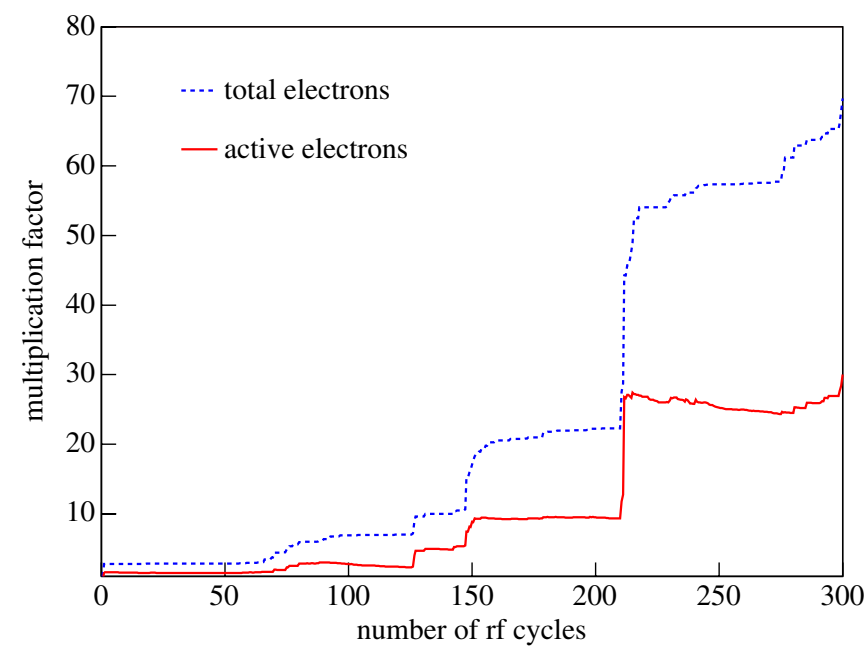

FIG. 19. (Color) Multiplication factor as a function of time over $300 \mathrm{rf}$ cycles while the rf field decays from $0.6 \mathrm{MV} / \mathrm{m}$ to about $0.55 \mathrm{MV} / \mathrm{m}$. The solenoid currents are $400 \mathrm{~A}$ for the main solenoid and $30.5 \mathrm{~A}$ for the bucking solenoid. rf cycles at the rf frequency of $1.3 \mathrm{GHz}$. The expansion of the secondary electron avalanche is limited by the decay of the rf field, and possibly also by beam loading from the multipacting electrons.

These numerical simulations do not completely explain the different multipacting behavior for the different solenoid profiles in the PITZ gun described in Sec. III D. In the real situation, the SEY of the $\mathrm{Cs}_{2} \mathrm{Te}$ cathode might vary with the rf field strength and the solenoid field strength applied to the $\mathrm{Cs}_{2} \mathrm{Te}$ film. Such effects were not included in the simulation study. Nevertheless, the general aspects of the multipacting could be reproduced by the numerical calculation with ASTRA.

\section{MULTIPACTING AT THE FNPL AND TTF1 GUNS}

The first photocathode rf gun in operation at the TESLA Test Facility Phase 1 (TTF1) [37] was a copy of the FNPL gun, while the new gun in operation at FLASH is a copy of the PITZ gun. In the design of the FNPL and TTF1 gun, the first focusing solenoid, referred to as the primary solenoid, is located closer to the cathode $(0.105 \mathrm{~m}$ downstream of the cathode) than in the PITZ design. This leads to a strong magnetic field gradient near the cathode and increases the strength of the magnetic mirror effect (compare Fig. 2 with Fig. 5). A simulation of multipacting in the FNPL/TTF1 gun was done with the three solenoid currents set to the same value, corresponding to a peak magnetic field of $0.12 \mathrm{~T}$. Figure 20 shows the multiplication factor as a function of the emission phase and the rf field at the cathode. The area in which multipacting can take place is much larger than in the PITZ and FLASH cases, and the strength of the multiplication is also greater.

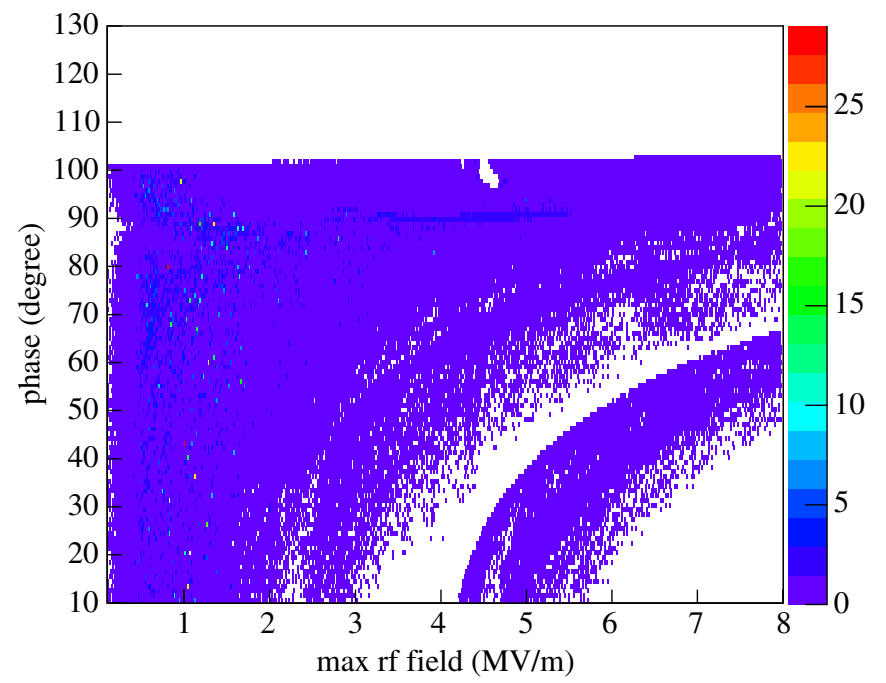

FIG. 20. (Color) Electron multiplication factor for the FNPL/ TTF1 gun. For this simulation, the currents in the three solenoids were set to be the same value (round beam settings), producing a peak magnetic field of 0.12 T. Primary and secondary electrons were tracked for $100 \mathrm{rf}$ cycles. The white area shows no multipacting condition. 


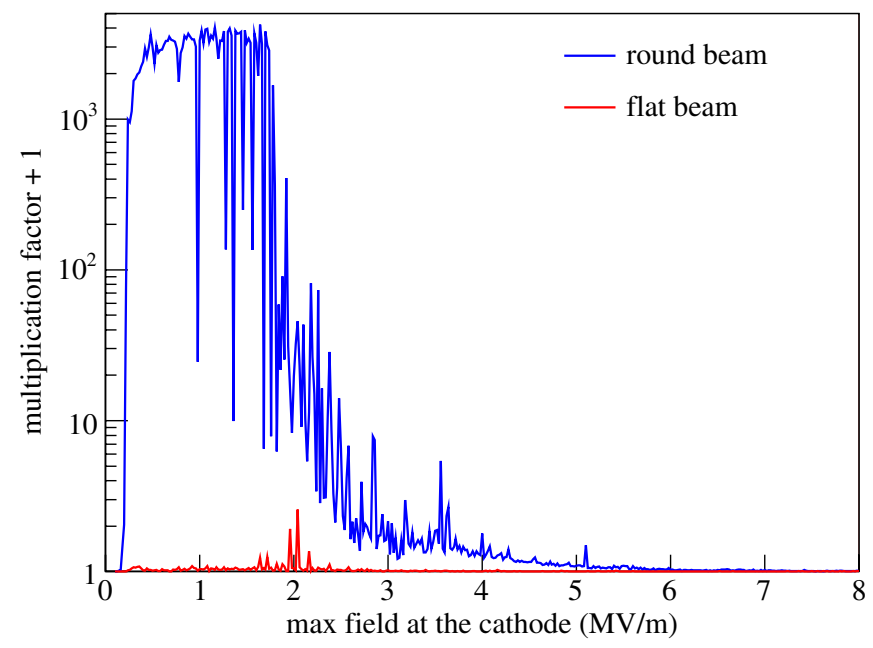

FIG. 21. (Color) Multiplication factor after $140 \mathrm{rf}$ cycles for the FNPL gun with two different solenoid configurations. In this simulation, 1000 seed particles were used and the secondary generation was limited to $6 \times 10^{6}$ particles. The multiplication factors between 0.5 and $1.6 \mathrm{MV} / \mathrm{m}$ are therefore underestimated for the round beam configuration. As before, 1 is added to the multiplication factor for visualization on a logarithmic scale.

Figure 21 compares the multiplication factors of the FNPL gun for round beam and flat beam solenoid settings. The multiplication factor for the round beam setting exceeds 1000 over a large range of maximum rf field. For the flat beam setting, the multiplication factor is much smaller than 1. This simulation result explains the observed multipacting behavior [6]. The multiplication for the FNPL gun with the round beam setting is 100 times stronger than the multiplication for the PITZ/FLASH gun with a main solenoid current of $400 \mathrm{~A}$ and a bucking solenoid current of 30.5 A. Time dependent variation in the dark current and QE dependent on the solenoid settings, i.e., the presence of the multipacting, has been observed clearly in the FNPL gun. This effect is likely related to the cleaning of the cathode surface by back bombardment from a huge number of secondary electrons. At the PITZ gun, the presence of the multipacting did not visibly change the cathode properties.

With the TTF1 gun, abnormally very high dark current values, as high as $3 \mathrm{~mA}$, were observed [38], sometimes with a drastic increase within days. Whether the abrupt increase is related to the strong multipacting is not clear. A similar behavior has not been observed at PITZ or FLASH. At the nominal operating conditions for both the TTF1 and FLASH guns, the measured dark current is typically lower than $0.3 \mathrm{~mA}$.

\section{DISCUSSION AND CONCLUSION}

In rf gun cavities with semiconductor, i.e. $\mathrm{Cs}_{2} \mathrm{Te}$, photocathodes, multipacting can take place at the cathode at low rf field, with the help of the magnetic field of the focusing solenoids. This single-side multipacting cannot be elimi- nated even with careful conditioning, because the cathode must have a high QE; in general, high QE materials have a high SEY. However, the multipacting may be controlled with a proper solenoid configuration.

Because the multipacting properties are related to the SEY of the cathode, the rf field amplitude at which the multipacting occurs and the charge in the multipacting peak may depend on the cathode preparation recipe and the cathode's surface condition. Nevertheless, the overall trends are consistent for measurements done at different times and with different cathodes.

This study will be useful to control multipacting in the design of photocathode rf guns, e.g., for the European $\mathrm{x}$-ray FEL [39], and in the design of secondary emission enhanced photoinjectors, a new type of photocathode gun combined with a secondary emitter [40,41]. On the one hand, it is preferable to avoid multipacting under nominal operating conditions for lower dark current and safe operation. On the other hand, multipacting can be helpful for possible cleaning of chemical pollution of the cathode surface to improve the QE under certain operating configurations $[5,6]$.

\section{ACKNOWLEDGMENTS}

The authors thank M. Krasilnikov and F. Stephan for helpful discussions and all PITZ members for their experimental support at DESY-Zeuthen, and for allowing beam time for these studies. We thank our FNPL collaborators who provided assistance and beam time for the experimental work at Fermilab. In particular, D. Finley and R. Fliller were valuable collaborators in the studies of field emission, photoemission, and multipacting. Essential expertise in photocathodes was provided by INFN-Milano. We would especially like to thank P. Michelato, L. Monaco, and D. Sertore for their support for both PITZ and FNPL.

[1] A. J. Hatch and H.B. Williams, Phys. Rev. 112, 681 (1958).

[2] U. Klein and D. Proch, in Proceedings of the Conference of Future Possibilities for Electron Accelerators, Charlottesville, VA, 1979, p. N1.

[3] J. R. M. Vaughan, IEEE Trans. Electron Devices 35, 1172 (1988).

[4] A. L. Gilardini, J. Appl. Phys. 78, 783 (1995).

[5] W. Hartung et al., in Proceedings of the 2001 Particle Accelerator Conference, Chicago, USA, 2001 (IEEE, Piscataway, NJ, 2001), p. 2239.

[6] W. Hartung et al. (unpublished).

[7] E. Somersalo, P. Ylä-Oijala, D. Proch, and J. Sarvas, Part. Accel. 59, 107 (1998).

[8] B. Henrist, N. Hilleret, C. Scheuerlein, M. Taborelli, and G. Vorlaufer, in Proceedings of the 2002 European Particle Accelerator Conference, Paris, France, 2002 (EPS-IGA and CERN, Geneva, 2002), p. 2553. 
[9] P. Michelato, Nucl. Instrum. Methods Phys. Res., Sect. A 393, 455 (1997).

[10] D. Sertore, P. Michelato, L. Monaco, S. Schreiber, J.-H. Han, and A. Bonucci, in Proceedings of the 2005 Particle Accelerator Conference, Knoxville, USA, 2005, p. 671.

[11] http://adweb.desy.de/pitz/web/.

[12] J.-H. Han, M. Krasilnikov, and K. Flöttmann, Phys. Rev. ST Accel. Beams 8, 033501 (2005).

[13] B. E. Carlsten, Nucl. Instrum. Methods Phys. Res., Sect. A 285, 313 (1989).

[14] R. Brinkmann, Y. Derbenev, and K. Flöttmann, Phys. Rev. ST Accel. Beams 4, 053501 (2001).

[15] Y.-E. Sun, P. Piot, K.-J. Kim, N. Barov, S. Lidia, J. Santucci, R. Tikhoplav, and J. Wennerberg, Phys. Rev. ST Accel. Beams 7, 123501 (2004).

[16] Y.-E. Sun, P. Piot, and K.-J. Kim, Phys. Rev. ST Accel. Beams 9, 031001 (2006).

[17] J.-H. Han et al., in Proceedings of the 2005 Particle Accelerator Conference, Knoxville, TN (IEEE, Piscataway, NJ, 2005), p. 895.

[18] J.H. Billen and L.M. Young, Los Alamos National Laboratory, Technical Report LA-UR-96-1834, 2000.

[19] A. Fry, E. Hahn, W. Hartung, M. Kuchnir, P. Michelato, and D. Sertore, in Proceedings of the XIX International Linac Conference, Chicago, 1998, p. 642.

[20] A. di Bona, F. Sabary, S. Valeri, P. Michelato, and D. Sertore, J. Appl. Phys. 80, 3024 (1996).

[21] R. P. Fliller, H. Edwards, and W. Hartung, in Proceedings of the 2005 Particle Accelerator Conference, Knoxvelle, TN (IEEE, Piscataway, NJ, 2005), p. 2681.

[22] CST MicrowaveStudio, CST GmbH, http://www.cst.de/.

[23] J.-H. Han, Ph.D. thesis, University of Hamburg, 2005 [DESY-Thesis-2005-038].

[24] Cathodes information available at http://wwwlasa.mi. infn.it/ttfcathodes/.
[25] S. Schreiber, P. Michelato, L. Monaco, and D. Sertore, in Proceedings of the 2003 Particle Accelerator Conference, Portland, OR (IEEE, Piscataway, NJ, 2003), p. 2071.

[26] L. Staykov et al., PITZ-Note, DESY, 2005.

[27] J.W. Wang and G.A. Loew, SLAC, Technical Report SLAC-PUB-7684, 1997.

[28] T. Thon and R. Wenndorff (private communication).

[29] S. Schreiber, P. Michelato, L. Monaco, D. Sertore, and J.-H. Han, in Proceedings of the 2004 European Particle Accelerator Conference, Lucerne, Switzerland (EPS-IGA and CERN, Geneva, 2004), p. 348.

[30] http://flash.desy.de/.

[31] A. H. Sommer, J. Appl. Phys. 42, 567 (1971).

[32] K. Flöttmann, ASTRA User's Manual, http://www. desy.de/ mpyflo/Astra_dokumentation.

[33] M. A. Furman and M.T.F. Pivi, Phys. Rev. ST Accel. Beams 5, 124404 (2002).

[34] J.D. Jackson, Classical Electrodynamics (Wiley, New York, 1998), 3rd ed.

[35] R. H. Fowler and L. Nordheim, Proc. R. Soc. A 119, 173 (1928).

[36] L. Nordheim, Proc. R. Soc. A 121, 626 (1928).

[37] V. Ayvazyan et al., Phys. Rev. Lett. 88, 104802 (2002).

[38] D. Sertore, S. Schreiber, F. Stephan, K. Zapfe, D. Hubert, and P. Michelato, DESY, Report No. TESLA-FEL 200005, 2000.

[39] http://xfel.desy.de/.

[40] I. Ben-Zvi, X. Chang, P. D. Johnson, J. Kewisch, and T. S. Rao, Accelerator Physics Note C-A/AP/149, BNL, 2004.

[41] X. Chang, I. Ben-Zvi, A. Burrill, J. Grimes, T. Rao, Z. Segalov, J. Smedley, and Q. Wu, in Proceedings of the 2007 Particle Accelerator Conference, Albuquerque, NM, 2007, p. 2044. 